\title{
SEA Antagonizes the Imatinib-Meditated Inhibitory Effects on T Cell Activation via the TCR Signaling Pathway
}

\author{
Guanming Wang, ${ }^{1,2}$ Yuhui Yan, ${ }^{1}$ Xiaohua Chen, ${ }^{1}$ Chen Lin, ${ }^{1}$ and Yangqiu Li ${ }^{3,4}$ \\ ${ }^{1}$ Department of Microbiology and Immunology, Medical College, Jinan University, Guangzhou 510632, China \\ ${ }^{2}$ Quanzhou Medical college, Quanzhou 362000, China \\ ${ }^{3}$ Institute of Hematology, Medical College, Jinan university, Guangzhou 510632, China \\ ${ }^{4}$ Key Laboratory for Regenerative Medicine of Ministry of Education, Guangzhou 510632, China
}

Correspondence should be addressed to Chen Lin; tlinc@jnu.edu.cn and Yangqiu Li; jnyangqiuli@163.com

Received 17 October 2013; Revised 26 November 2013; Accepted 10 December 2013; Published 2 January 2014

Academic Editor: Masahisa Jinushi

Copyright (C) 2014 Guanming Wang et al. This is an open access article distributed under the Creative Commons Attribution License, which permits unrestricted use, distribution, and reproduction in any medium, provided the original work is properly cited.

\begin{abstract}
The BCR-ABL kinase inhibitor imatinib is highly effective in the treatment of chronic myeloid leukemia (CML). However, long-term imatinib treatment induces immunosuppression, which is mainly due to T cell dysfunction. Imatinib can reduce TCR-triggered $\mathrm{T}$ cell activation by inhibiting the phosphorylation of tyrosine kinases such as Lck, ZAP70, LAT, and PLC $\gamma 1$ early in the TCR signaling pathway. The purpose of this study was to investigate whether the superantigen SEA, a potent T cell stimulator, can block the immunosuppressive effects of imatinib on T cells. Our data show that the exposure of primary human T cells and Jurkat cells to SEA for $24 \mathrm{~h}$ leads to the upregulation of the Lck and ZAP70 proteins in a dose-dependent manner. T cells treated with SEA prior to TCR binding had increased the tyrosine phosphorylation of Lck, ZAP70, and PLC $\gamma 1$. Pretreatment with SEA prevents the inhibitory effects of imatinib on TCR signaling, which leads to T cell proliferation and IL-2 production. It is conceivable that SEA antagonizes the imatinib-mediated inhibition of $\mathrm{T}$ cell activation and proliferation through the TCR signaling pathway.
\end{abstract}

\section{Introduction}

Imatinib (IM; formerly STI571) is a tyrosine kinase inhibitor that has strong activity against the BCR/ABL tyrosine kinase, a fusion protein that causes the onset of Philadelphia chromosome positive chronic myeloid leukemia (CML) and broad activity against Src-family tyrosine kinases, such as c-KIT and platelet-derived growth factor receptor, which play a role in gastrointestinal stromal tumors. The introduction of IM for the treatment of CML represents an ideal example of molecular targeted therapy in human cancer. It is also conceivable that although imatinib is a successful kinase inhibitor in clinical use, immunosuppression, an imatinibassociated side effect, is inevitable. Accumulating evidence shows that long-term imatinib treatment induces defects in humoral and cell-mediated immunity [1-5].

A new generation of tyrosine kinase targeted drugs, such as dasatinib and nilotinib, are effective in many cases in which disease is resistant to imatinib. These drugs have been reported to inhibit $\mathrm{T}$ cell function in vitro, in mouse models [6-13], and in CML patients $[14,15]$.

Studies have shown that the inhibition of Lck and other kinases involved in $\mathrm{T}$ cell signaling by tyrosine kinase inhibitors, including imatinib, dasatinib, and nilotinib, is responsible for the suppression of $\mathrm{T}$ cell function. The Src kinase Lck is a key target of these compounds during T cell activation. These findings raise a concern for potential $\mathrm{T}$ cell inhibition in patients taking tyrosine kinase inhibitors. While targeted therapy could induce CML remission, this disease remains largely incurable. We have come to realize that induction of the immune response may lead to a cure for this disease [16-21].

$\mathrm{T}$ cells play a central role in cell immunity. The $\mathrm{T}$ cell receptor (TCR) signaling cascade is initiated by engagement of antigenic peptides bound to the major histocompatibility complex (MHC). Upon TCR stimulation, CD4/CD8-associated Lck is brought into proximity with 
TCR/CD3 complexes and phosphorylates the immunoreceptor tyrosine-based activation motifs (ITAMs) of the CD3 subunits. ZAP70 is then recruited to the phosphorylated ITAMs and subsequently activated by Lck. Activated ZAP70 phosphorylates LAT, which in turn phosphorylates PLC $\gamma 1$ and other signaling molecules, resulting in the activation of multiple pathways. These sequential molecular events eventually lead to $\mathrm{T}$ cell activation and IL-2 production.

Although the mechanism of imatinib-mediated TCR signaling inhibition has been well defined, a feasible solution for imatinib-mediated immunosuppression is unknown. As the critical tyrosine kinase in the TCR signaling pathway, Lck is vital for $\mathrm{T}$ cell activation. In Lck-deficient $\mathrm{T}$ cell lines, T cells fail to induce ZAP70 phosphorylation and $\mathrm{Ca}^{+}$ mobilization following TCR stimulation. On this basis, Lck inhibition by imatinib due to its sequence homology with ABL kinase in the adenosine triphosphate (ATP) binding pocket consequentially results in decreased TCR-mediated $\mathrm{T}$ cell activation [22]. It has been demonstrated that Lck determines the threshold for T cell activation and a lack of Lck in T cells leads to an increased activation threshold [23-25].

Because Lck expression affects T cell activation and proliferation after TCR engagement, it is reasonable to hypothesize that the Lck upregulated during imatinib treatment decreases the threshold of $\mathrm{T}$ cell activation and to some extent may enhance $\mathrm{T}$ cell activation, proliferation, and cytokine production. Unlike conventional peptide antigens, bacterial superantigens (SAgs) are protein toxins that bind to the external surfaces of the TCR and MHC class II to simultaneously activate large numbers of T cells [26]. Therefore, SAgs have been considered to activate $T$ cells through the canonical signaling pathway, which includes TCR engagement of peptide-MHC complexes. Previous findings have shown that the superantigen staphylococcal enterotoxin A (SEA) not only powerfully activates $\mathrm{T}$ cells, but also induces broad expression of genes related to cytokine production and TCR signal transduction [27-29], implying that SEA may affect the expression of early kinases in the TCR signaling pathway. The aim of this study was to investigate the effect of SEA on the expression of Lck, Fyn, and ZAP70 and determine whether this effect could antagonize the imatinib-mediated inhibition of $\mathrm{T}$ cell activation. We demonstrate that SEA upregulates the expression and phosphorylation of Lck and subsequently avoids the imatinib-mediated inhibition of T cell activation.

\section{Materials and Methods}

2.1. Cell Preparation, Chemicals, and Antibodies. The Jurkat cell line was obtained from American Type Culture Collection (ATCC). Peripheral blood mononuclear cells (PBMCs) were obtained from four healthy donors who provided informed consent. Mononuclear cells were isolated by Ficolldiatrizoate density gradient centrifugation (Sigma Chemical Co., USA). Imatinib mesylate (Glivec, STI571) was purchased from Selleck Chemicals (USA), and SEA was obtained from Sigma (USA). The following antibodies were used as primary antibodies : rabbit monoclonal anti-Lck, anti-Fyn, and antiZAP70 (Epitomics, USA), mouse monoclonal anti-GAPDH
(Santa Cruz Biotechnology, USA), mouse monoclonal antiphospho-Lck (Tyr394) (GeneTex, USA), rabbit monoclonal anti-phospho-ZAP70 (Tyr319) (Cell Signaling Technology, USA), and rabbit monoclonal anti-phospho-PLC $\gamma 1$ (Tyr783) (Epitomics, USA). Secondary antibodies were purchased from Santa Cruz Biotechnology (USA).

2.2. Cell Culture. Jurkat cells were maintained in RPMI 1640 medium (Gibco, USA) supplemented with $10 \%$ fetal bovine serum (Hangzhou Sijiqing Company, China), $100 \mathrm{U} / \mathrm{mL}$ penicillin, and $100 \mu \mathrm{g} / \mathrm{mL}$ streptomycin (Invitrogen) at $37^{\circ} \mathrm{C}$ in a humidified incubator with $5 \% \mathrm{CO}_{2}$. Culture media was passaged every 2-3 days.

2.3. ELISA for $I L-2$. Jurkat cells or human PBMCs $(1 \times$ $10^{5} /$ well) in a total volume of $200 \mu \mathrm{L}$ were incubated in 96 well plates in the presence or absence of SEA $(20 \mathrm{ng} / \mathrm{mL})$ for $24 \mathrm{~h}$ and subsequently washed with PBS. Afterward, cells were treated with or without imatinib $(40 \mathrm{nM})$ for $1 \mathrm{~h}$ followed by stimulation with anti-CD3/CD28 coated beads at a cell: bead ratio of $5: 1$ for $24 \mathrm{~h}$. The IL- 2 content in the supernatant was measured with an ELISA kit (RayBiotech, USA) following the manufacturer's protocol.

2.4. Cell Proliferation Assay. Cells were treated the same as above. After treatment, $10 \mu \mathrm{L}$ of CCK- 8 solution (Dojindo, Japan) was added into each well, and the cells were incubated at $37^{\circ} \mathrm{C}$ for $2 \mathrm{~h}$. The sample absorbance was then recorded at $450 \mathrm{~nm}$.

2.5. Western Blotting Analysis. Jurkat cells $\left(3 \times 10^{6} /\right.$ well $)$ were cultured in 6-well plates with different concentrations of SEA for $24 \mathrm{~h}$. Cells without treatment served as a negative control. The expression of Lck, Fyn, and ZAP70 was examined by Western blotting [7]. Briefly, cells were lysed in $1 \mathrm{~mL}$ of lysis buffer containing 1\% Triton X-100. Protein concentrations were determined by the Bradford assay. The proteins were separated by electrophoresis in a $10 \%$ SDS-polyacrylamide gel and transferred onto a PVDF membrane. The blots were blocked with $5 \%(\mathrm{w} / \mathrm{v})$ nonfat dry milk constituted in $1 \mathrm{x}$ TBS$\mathrm{T}$ for $1 \mathrm{~h}$ at room temperature. Membranes were incubated at $4^{\circ} \mathrm{C}$ overnight with a primary monoclonal antibody in $5 \%$ bovine serum albumin (BSA) in 1x TBS-T and then with the respective horseradish peroxidase-conjugated secondary antibody as directed by the manufacturer for 1 hour at room temperature. Immunoreactive bands were visualized using the enhanced chemiluminescence light (ECL) detection reagent.

To assess the effects of SEA on the imatinib-mediated inhibition of the TCR signaling pathway, Jurkat cells and PBMCs $\left(3 \times 10^{6} /\right.$ well $)$ were pretreated with and without SEA $(20 \mathrm{ng} / \mathrm{mL})$ for $24 \mathrm{~h}$ followed by imatinib or control stimulation (40 nM) for 1 hour. Finally, cells were stimulated with anti-CD3 and anti-CD28 coated Dynabeads (Dynal, Invitrogen, Oslo, Norway) at a cell : bead ratio of 1: 1 for $15 \mathrm{~min}$ [28]. Whole-cell lysates were subjected to Western blot analysis to examine the phosphorylation level of Lck, ZAP70, and PLC $\gamma 1$ 


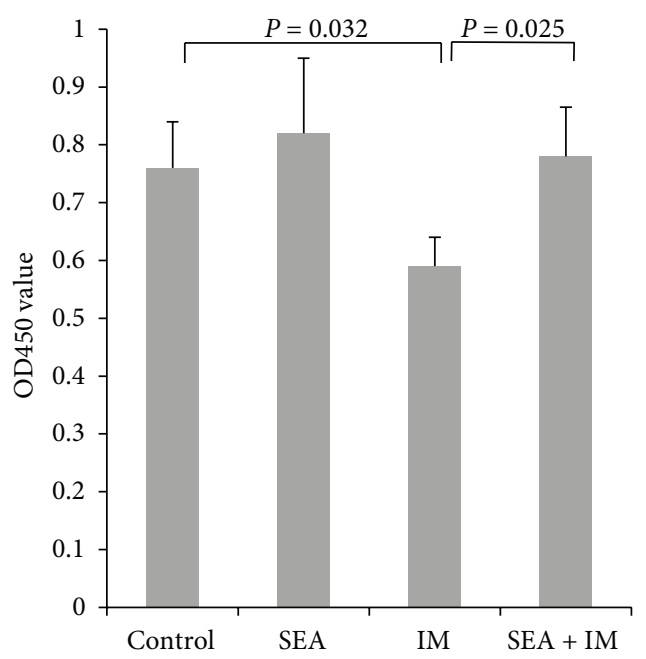

(a) Jurkat cells

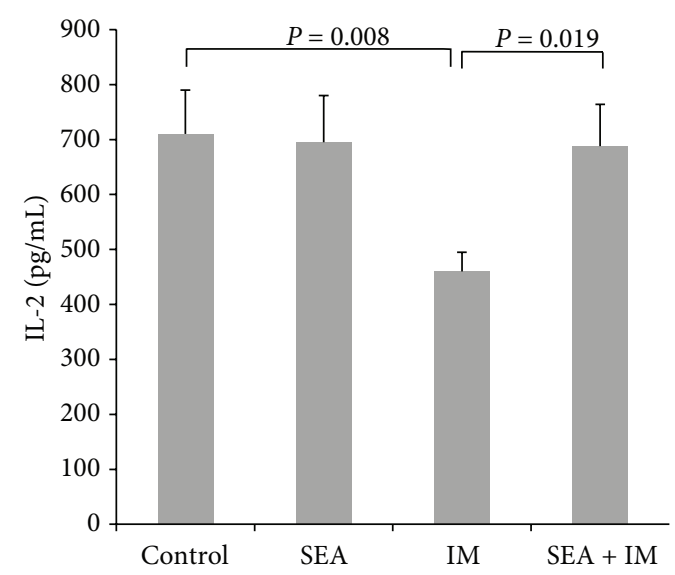

(c) Jurkat cells

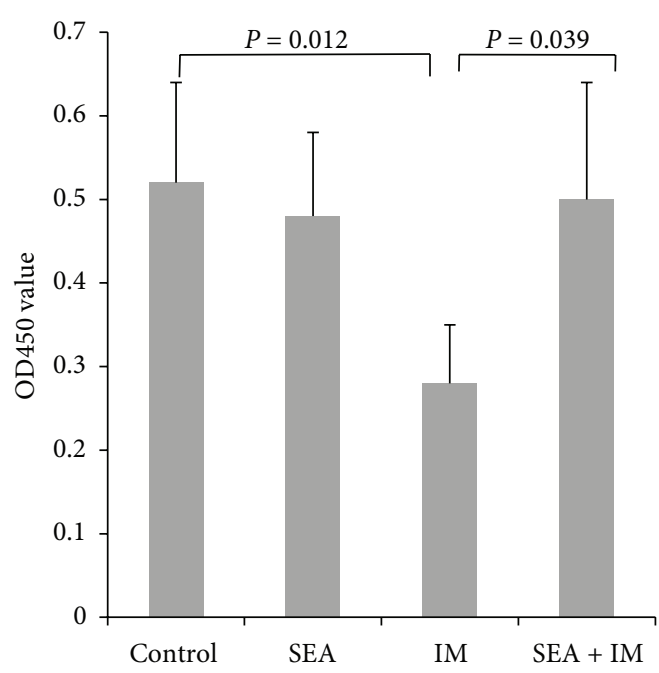

(b) PBMCs

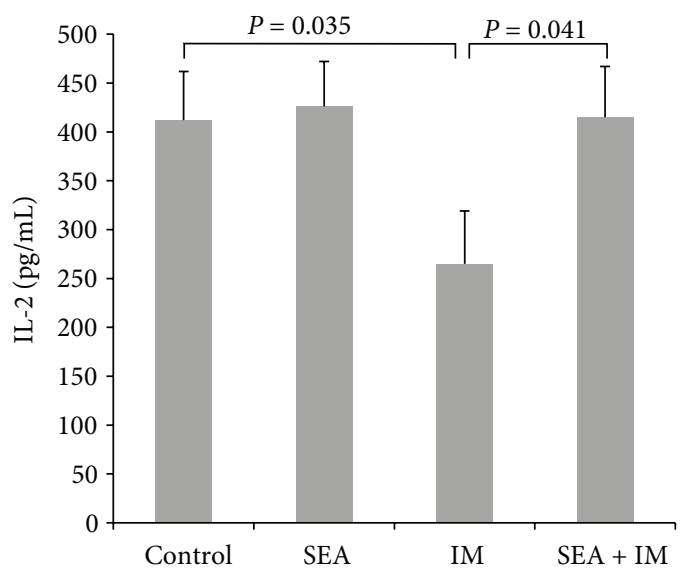

(d) PBMCs

FIGURE 1: The cell proliferation and IL-2 production of Jurkat cells and PBMCs following TCR stimulation. (Control) Untreated Jurkat cells or PBMCs. (SEA) Cells were pretreated with SEA $(20 \mathrm{ng} / \mathrm{mL}$ ). (IM) Cells were pretreated with $40 \mathrm{nM}$ imatinib. (SEA + IM) Cells were pretreated with SEA $(20 \mathrm{ng} / \mathrm{mL})$ for $24 \mathrm{~h}$ followed by $40 \mathrm{nM}$ imatinib treatment for $15 \mathrm{~min}$. Each group was stimulated with anti-CD3/CD28 coated beads at a cell : bead ratio of $5: 1$ for $24 \mathrm{~h}$. Cell proliferation was assayed with the CCK-8 kit (a) and (b). The IL-2 level was assayed using a human IL-2 ELISA kit (c) and (d). The mean value and standard deviation of 3 independent experiments are shown.

with the respective horseradish peroxidase-conjugated secondary antibody as directed by the manufacturer.

2.6. Statistical Analysis. The data were analyzed using SPSS version 16.0 statistical software. Error bars indicate SDs from the mean of at least three replicates. Statistical tests were conducted using the Student's test or analysis of variance (ANOVA) where indicated.

\section{Results}

3.1. SEA Reduces the Inhibitory Effects of Imatinib on T Cell Proliferation and IL-2 Production. It is known that imatinib suppresses $\mathrm{T}$ cell activation and proliferation. To investigate the effects of SEA on the inhibitory effects of imatinib on $\mathrm{T}$ cell proliferation and IL-2 production, Jurkat cells and PBMCs were preincubated with SEA and then washed followed by treatment with imatinib and stimulation with anti-CD3/CD28 coated beads. T cell proliferation and IL-2 production were measured using the CCK-8 kit and an IL2 ELISA kit. The T cell proliferation and the IL-2 level were significantly decreased with imatinib treatment alone, but this suppression could be reversed with SEA pretreatment $(P<0.05)$ (Figure 1).

3.2. SEA Upregulates Lck and ZAP70 in a Dose-Dependent Manner. The effects of SEA on the imatinib-mediated inhibition of $\mathrm{T}$ cell activation and proliferation could be due to the upregulation of proximal signaling events mediated by the TCR because imatinib could inhibit TCR signal transduction [7]. Therefore, we used the well-characterized Jurkat $\mathrm{T}$ cell line to determine the level of signaling protein expression in response to SEA. 


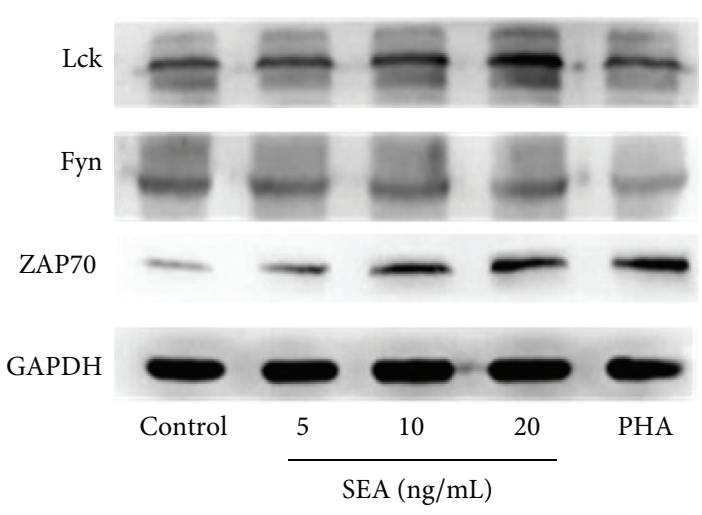

(a)

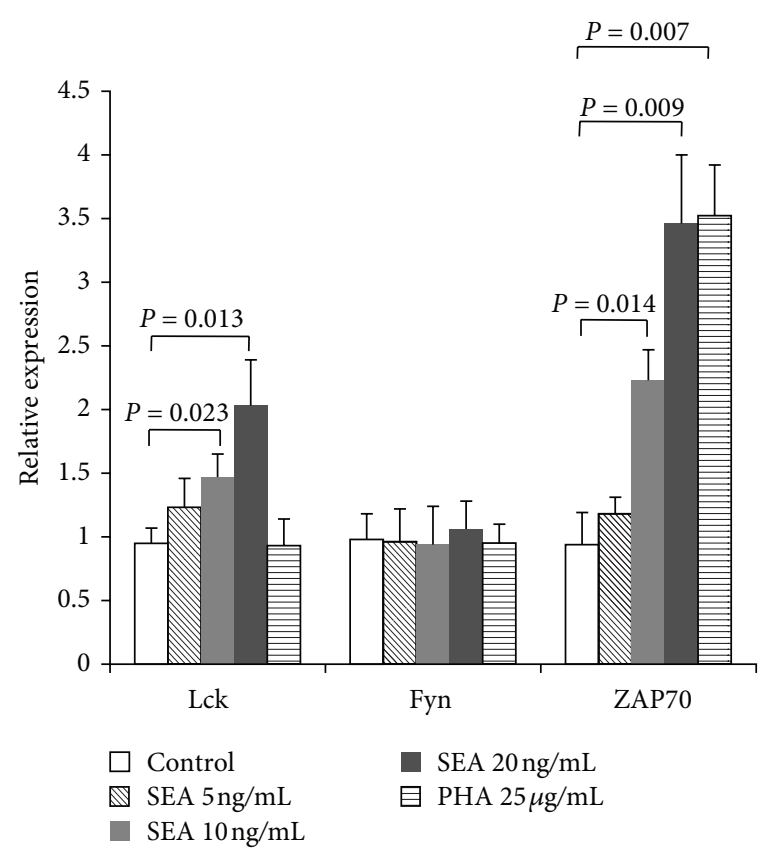

(b)

FIGURE 2: SEA increases Lck and ZAP70 expression in Jurkat cells. (a) Western blot analysis of Lck, Fyn, and ZAP70 after $24 \mathrm{~h}$ of incubation with the indicated concentrations of SEA. GAPDH served as a loading control. (b) Densitometry (target protein : GAPDH ratio normalized to control) was conducted on three independent experiments and is depicted in the bar graph.

Jurkat cells were incubated with different concentrations of SEA ranging from 5 to $20 \mathrm{ng} / \mathrm{mL}$ for $24 \mathrm{~h}$, and cell lysates were subjected to Western blot analysis. The results demonstrated that SEA increases Lck and ZAP70 protein expression in a dose-dependent manner, but there was no effect on Fyn expression (Figure 2).

3.3. SEA Abrogates the Inhibitory Effects of Imatinib on TCR Signaling. It has been reported that imatinib selectively inhibits Lck and its downstream signaling molecules [7]. Given the restricted expression of Lck, which binds to CD $4 / 8$ and is located within the vicinity of TCR-CD3 complex, as a superantigen, SEA not only causes upregulation of the Lck and ZAP70 proteins but also might cause an increase in the phosphorylation of Lck and downstream proteins by binding the TCR V beta chain. We asked whether the upregulation of Lck by SEA could prevent the suppressive effects of imatinib during $\mathrm{T}$ cell activation. To test this hypothesis, we measured the phosphorylation of Lck and its downstream molecules during $\mathrm{T}$ cell stimulation. We found that the Lck, ZAP70, and PLC $\gamma 1$ phosphorylation in T cells, including the Jurkat cell line and PBMCs, could be increased by SEA and the reduction in the activating phosphorylations of these four signaling proteins caused by imatinib was prevented after pretreatment with SEA followed by TCR stimulation (Figure 3). SEA rescues $\mathrm{T}$ cells from inhibition by imatinib.

\section{Discussion}

Small molecule tyrosine kinase inhibitors have been successfully used for the treatment of CML. Imatinib and dasatinib are two of the most commonly used tyrosine kinase inhibitors, and both have been shown to impact $\mathrm{T}$ cell function. Imatinib inhibits $\mathrm{T}$ cell receptor-mediated $\mathrm{T}$ cell activation and proliferation in a dose-dependent manner $[1,7]$. Brauer et al. described the downregulation of several LAAs in CMLs treated with imatinib [30]. Due to this activity, their use as potential immunosuppressants has been proposed. These agents have been recently used to treat autoimmune diseases, such as immune-mediated kidney injury and rheumatoid arthritis. Tyrosine kinase inhibitors (TKIs) are effective for the treatment of these diseases [31,32].

With the development and use of TKIs being rapidly expanding, their potential side effects against cancer are of considerable clinical importance. The T cell suppressive effects of imatinib and dasatinib have been attributed to Lck inhibition. Our study is consistent with published results demonstrating that imatinib perturbs TCR signaling, inhibiting the phosphorylation of LCK and Zap70 following TCR stimulation (Figure 3).

Lck, a src family kinase, is critical for $\mathrm{T}$ cell activation, and its activity is tightly regulated in lipid rafts. Indeed, an appropriate balance between active and inactive Lck is essential for safe TCR sensitivity, and abnormal T cell responsiveness occurs if the balance is broken. On one hand, an absence or reduction in Lck activity leads to an impairment in the activation of mature $\mathrm{T}$ cells. On the other hand, increased Lck phosphorylation is associated with $\mathrm{T}$ cell hyperresponsiveness, which can induce autoimmune disease such as that exhibited by SLE patients [33, 34]. With respect to the role of Lck in $\mathrm{T}$ cell activation, Lovatt et al. support the suggestion that Lck sets a threshold of 


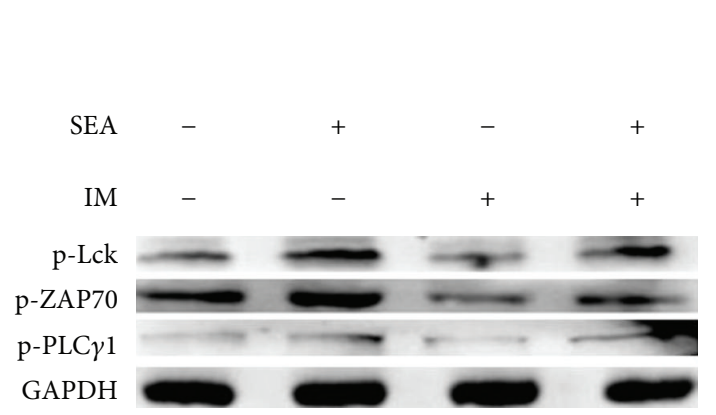

(a) Jurkat cells

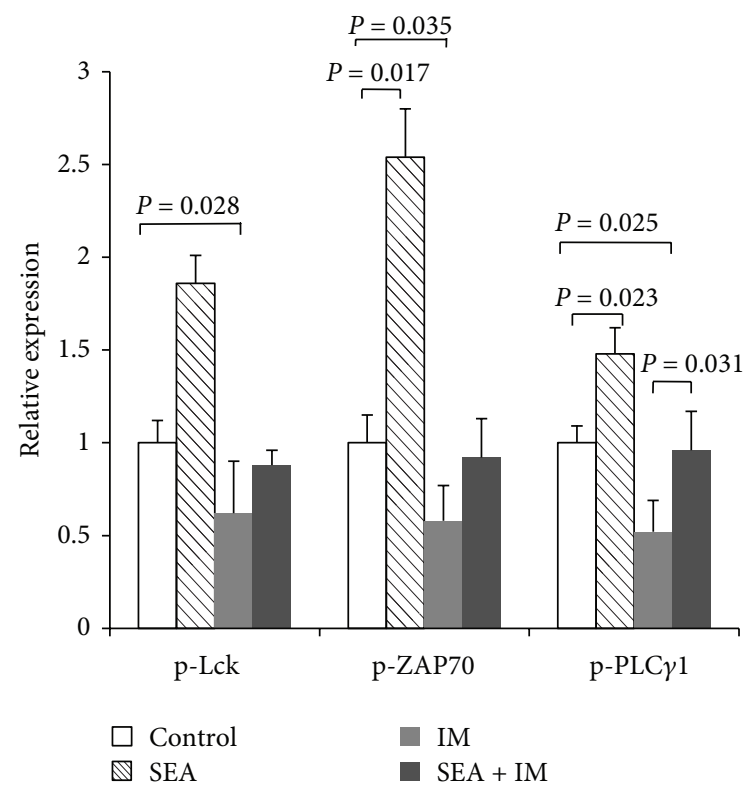

(c) Jurkat cells

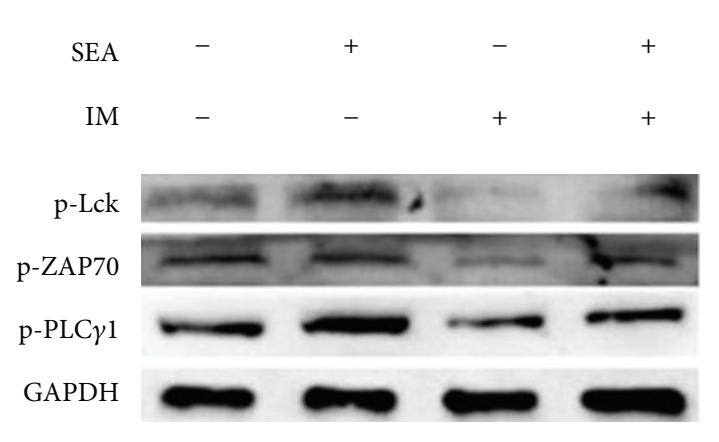

(b) PBMCs

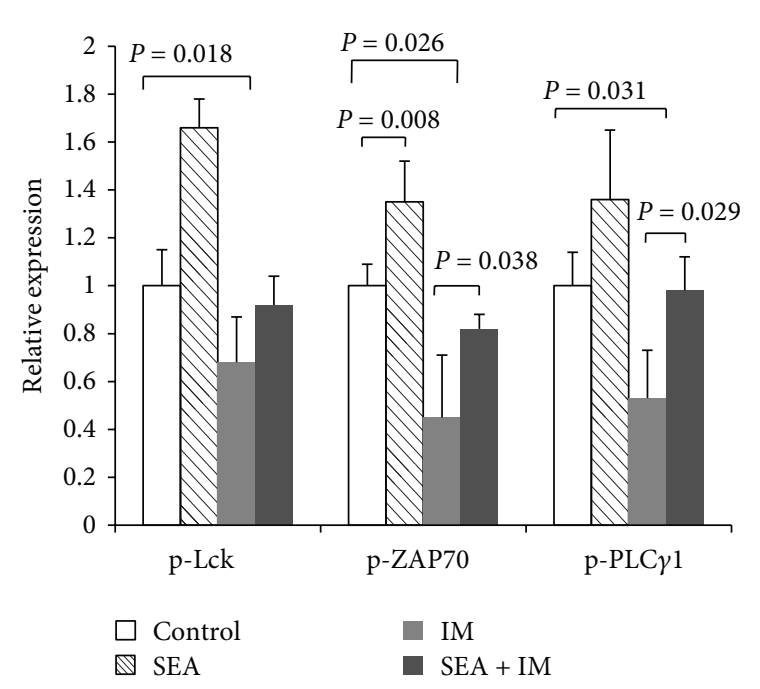

(d) PBMCs

FIGURE 3: SEA pretreatment inhibited the reduction in imatinib-induced phosphorylation of Lck, ZAP70, and PLC $\gamma 1$ in Jurkat cells (a) and PBMCs (b). Densitometry (target protein : GAPDH ratio normalized to control) was conducted on three independent experiments in Jurkat cells (c) and PBMCs (d). Jurkat cells or PBMCs were pretreated in the absence or presence of SEA ( $20 \mathrm{ng} / \mathrm{mL})$ followed by treatment with or without imatinib $(40 \mathrm{nM})$, and cells were then stimulated with anti-CD3/CD28 coated beads for $15 \mathrm{~min}$. Total cell extracts were resolved by SDS-PAGE and subjected to immunoblot analysis using phosphospecific antibodies as indicated.

activation in T cells [24]. Accordingly, Lck is undoubtedly a key protein in the strategy of eliminating imatinib-induced immunosuppression.

Effective biologic treatment is a promising solution for tumors. In particular, the existence of a suppressed immune response directed against tumors can be relieved by treatment, or, alternatively, treatment needs to be combined with an immune-enhancing therapy. In general, bacterial SAgs can simultaneously interact with MHC II and TCR molecules to activate $\mathrm{T}$ cells, which produce cytokines such as IL-2 and TNF- $\alpha$ and both directly and indirectly enhance immunotherapy efficiency [35-37]. However, there were reports that superantigens can mediate T-cell-dependent killing of tumor cells independently of MHC class II molecule expression on the target cell and the Asp227Ala replacement was introduced to destroy the site having the highest affinity for MHC class II in SEA to decrease the reactivity of the superantigen with MHC class II bearing cells for the treatment of human colorectal cancer [38-40]. These studies indicated that a direct superantigen-TCR interaction could result in superantigens activation of T cells in a MHC class II-free system. And these findings could support our results that SEA could directly stimulate Jurkat cells.

Bacterial SAgs cause polyclonal $\mathrm{T}$ cell activation due to their capacity to bind different TCR V $\beta$ chains [41-43]. Jurkat cells are originally from TCR V $\beta 8$ T cell clone. And the $\mathrm{V} \beta 8$ chain could react with SEA weakly [44]. Lck is activated after TCR engagement with SAgs [45]. Dauwalder et al. studied the early kinetics of the transcriptional response of PBMCs primed with SEA and SEG. These authors concluded that SEA, in contrast with SEG, induced the early transcriptional activation of several pathways, including $\mathrm{T}$ cell activation mediators and TCR-mediated signaling [29]. Although several reports have shown that bacterial SAgs can activate human $\mathrm{T}$ cells lacking Lck, suggesting the existence of an additional TCR signaling pathway [46, 47], in this study, we demonstrated that SEA could upregulate Lck and ZAP70 expression and phosphorylation following TCR 
stimulation. SEA has shown effects that are antagonistic to the imatinib-mediated inhibition of Lck phosphorylation and downstream signaling molecules after T cells were pretreated with SEA (Figure 3). This antagonistic effect may be due to an increase in Lck and ZAP70 expression. Because the imatinibinduced inhibition of Lck phosphorylation occurs in a dosedependent manner [7], the amount of phosphorylated Lck reduction is limited at a certain concentration of imatinib. Thus, Lck is able to trigger TCR-mediated expansion signals if basal Lck expression is increased prior to imatinib treatment.

In contrast with the conventional paradigm for $\mathrm{T}$ cell activation in which Lck plays a critical activating role in the TCR signaling process, Criado and Madrenas reported that Lck is dispensable for $\mathrm{T}$ cell activation by SAgs, but it actively inhibits this signaling pathway. The disruption of Lck function led to increased IL-2 production in response to SAgs stimulation [48]. These observations could be explained by the multistage role of Lck in $\mathrm{T}$ cell activation signaling based on the two pools of Lck detected at the immunological synapse, that is, the central pool and the peripheral pool. Lck may have a dual role where, early in TCR engagement, the central Lck pool participates in the initiation and activation of downstream signaling molecules and the peripheral Lck pool downregulates TCR-dependent signaling [49].

In conclusion, although the immunomodulatory effects of imatinib and dasatinib remain controversial [50], it has been confirmed that increasing effective and specific immunotherapies involving vaccination or adoptive cellular immunotherapy are necessary for CML. In this study, we showed that imatinib may affect TCR-mediated immune responses and characterized the effects of SEA on the imatinib-mediated inhibition of $\mathrm{T}$ cell reactivation in which this superantigen increased the tyrosine phosphorylation of Lck, ZAP70, and PLC $\gamma$ in T cells. This finding suggests that the SEA could be used for the prevention of imatinibmediated $\mathrm{T}$ cell immunosuppression. In addition, increasing Lck upregulation is a feasible option for restimulating the immune response in CML patients with TKI treatment.

\section{Conflict of Interests}

No potential conflict of interests was disclosed.

\section{Authors' Contribution}

Chen Lin and Yangqiu Li contributed to the conception as well as the design and paper writing. Guanming Wang, Yuhui Yan, and Xiaohua Chen provided the study materials and methods besides collecting and assembling the data. Moreover, Guanming Wang and Chen Lin undertook the data analysis and interpretation. All authors read and approved the final paper.

\section{Acknowledgments}

The study was supported by Grants from the National Natural Science Foundation of China (no. 81270604), the Natural Science Foundation of Guangdong Province, China (no. S2013020012863), and the Fundamental Research Funds for the Central Universities (no. 21612116).

\section{References}

[1] K. Cwynarksi, R. Laylor, E. Macchiarulo et al., "Imatinib inhibits the activation and proliferation of normal T lymphocytes in vitro," Leukemia, vol. 18, no. 8, pp. 1332-1339, 2004.

[2] A. B. Dietz, L. Souan, G. J. Knutson, P. A. Bulur, M. R. Litzow, and S. Vuk-Pavlović, "Imatinib mesylate inhibits T-cell proliferation in vitro and delayed-type hypersensitivity in vivo," Blood, vol. 104, no. 4, pp. 1094-1099, 2004.

[3] H. Gao, B. N. Lee, M. Talpaz et al., "Imatinib mesylate suppresses cytokine synthesis by activated CD4 T cells of patients with chronic myelogenous leukemia," Leukemia, vol. 19, no. 11, pp. 1905-1911, 2005.

[4] G. N. Mattiuzzi, J. E. Cortes, M. Talpaz et al., "Development of Varicella-Zoster virus infection in patients with chronic myelogenous leukemia treated with imatinib mesylate," Clinical Cancer Research, vol. 9, no. 3, pp. 976-980, 2003.

[5] J. L. Steegmann, G. Moreno, C. Aláez et al., "Chronic myeloid leukemia patients resistant to or intolerant of interferon $\alpha$ and subsequently treated with imatinib show reduced immunoglobulin levels and hypogammaglobulinemia," Haematologica, vol. 88, no. 7, pp. 762-768, 2003.

[6] A. E. Schade, G. L. Schieven, R. Townsend et al., "Dasatinib, a small-molecule protein tyrosine kinase inhibitor, inhibits T-cell activation and proliferation," Blood, vol. 111, no. 3, pp. 1366-1377, 2008.

[7] R. Seggewiss, K. Loré, E. Greiner et al., "Imatinib inhibits T-cell receptor-mediated T-cell proliferation and activation in a dosedependent manner," Blood, vol. 105, no. 6, pp. 2473-2479, 2005.

[8] S. J. Blake, A. B. Lyons, and T. P. Hughes, "Nilotinib inhibits the Src-family kinase LCK and T-cell function in vitro," Journal of Cellular and Molecular Medicine, vol. 13, no. 3, pp. 599-601, 2009.

[9] J. Chen, A. Schmitt, B. Chen et al., "Nilotinib hampers the proliferation and function of CD8+ T lymphocytes through inhibition of T cell receptor signalling," Journal of Cellular and Molecular Medicine, vol. 12, no. 5, pp. 2107-2118, 2008.

[10] S. Blake, T. P. Hughes, G. Mayrhofer, and A. B. Lyons, "The Src/ ABL kinase inhibitor dasatinib (BMS-354825) inhibits function of normal human T-lymphocytes in vitro," Clinical Immunology, vol. 127, no. 3, pp. 330-339, 2008.

[11] C. K. Fraser, S. J. Blake, K. R. Diener et al., "Dasatinib inhibits recombinant viral antigen-specific murine CD4+ and CD8+ Tcell responses and NK-cell cytolytic activity in vitro and in vivo," Experimental Hematology, vol. 37, no. 2, pp. 256-265, 2009.

[12] F. Fei, Y. Yu, A. Schmitt et al., "Dasatinib exerts an immunosuppressive effect on CD8+ T cells specific for viral and leukemia antigens," Experimental Hematology, vol. 36, no. 10, pp. 12971308,2008

[13] R. Weichsel, C. Dix, L. Wooldridge et al., "Profound inhibition of antigen-specific T-cell effector functions by dasatinib," Clinical Cancer Research, vol. 14, no. 8, pp. 2484-2491, 2008.

[14] C. Sillaber, H. Herrmann, K. Bennett et al., "Immunosuppression and atypical infections in CML patients treated with dasatinib at $140 \mathrm{mg}$ daily," European Journal of Clinical Investigation, vol. 39, no. 12, pp. 1098-1109, 2009. 
[15] H. A. Torres and R. F. Chemaly, "Viral infection or reactivation in patients during treatment with dasatinib: a call for screening?" Leukemia and Lymphoma, vol. 48, no. 12, pp. 2308-2309, 2007.

[16] A. S. M. Yong, K. Keyvanfar, R. Eniafe et al., "Hematopoietic stem cells and progenitors of chronic myeloid leukemia express leukemia-associated antigens: implications for the graft-versusleukemia effect and peptide vaccine-based immunotherapy," Leukemia, vol. 22, no. 9, pp. 1721-1727, 2008.

[17] N. Ali, S. N. Adil, M. U. Shaikh et al., "Outcome of match related allogeneic stem cell transplantation procedures performed from 2004 till 2011," Experimental Hematology \& Oncology, vol. 1, no. 1, article 13, 2012.

[18] K. Lu and X. Wang, "Therapeutic advancement of chronic lymphocytic leukemia,", Journal of Hematology \& Oncology, vol. 5, article 55, 2012.

[19] X. Zha, S. Chen, L. Yang et al., "Upregulated TCR $\zeta$ enhances interleukin-2 production in T-cells from patients with CML," DNA Cell Biolog, vol. 31, no. 11, pp. 1628-1635, 2012.

[20] Y. Qin, H. Tian, G. Wang, C. Lin, and Y. Li, "A BCR/ABL-hIL-2 DNA vaccine enhances the immune responses in BALB/c mice," BioMed Research International, vol. 2013, Article ID 136492, 9 pages, 2013.

[21] K. Liseth, E. Ersvr, T. Hervig, and Ø. Bruserud, "Combination of intensive chemotherapy and anticancer vaccines in the treatment of human malignancies: the hematological experience," Journal of Biomedicine and Biotechnology, vol. 2010, Article ID 692097, 15 pages, 2010.

[22] B. Nagar, W. G. Bornmann, P. Pellicena et al., "Crystal structures of the kinase domain of c-Abl in complex with the small molecule inhibitors PD173955 and imatinib (STI-571)," Cancer Research, vol. 62, no. 15, pp. 4236-4243, 2002.

[23] R. J. Salmond, A. Filby, I. Qureshi, S. Caserta, and R. Zamoyska, "T-cell receptor proximal signaling via the Src-family kinases, Lck and Fyn, influences T-cell activation, differentiation, and tolerance," Immunological Reviews, vol. 228, no. 1, pp. 9-22, 2009.

[24] M. Lovatt, A. Filby, V. Parravicini, G. Werlen, E. Palmer, and R. Zamoyska, "Lck regulates the threshold of activation in primary T cells, while both Lck and Fyn contribute to the magnitude of the extracellular signal-related kinase response," Molecular and Cellular Biology, vol. 26, no. 22, pp. 8655-8665, 2006.

[25] S. Pryshchep, J. J. Goronzy, S. Parashar, and C. M. Weyand, "Insufficient deactivation of the protein tyrosine kinase lck amplifies t-cell responsiveness in acute coronary syndrome," Circulation Research, vol. 106, no. 4, pp. 769-778, 2010.

[26] J. D. Fraser and T. Proft, "The bacterial superantigen and superantigen-like proteins," Immunological Reviews, vol. 225, no. 1, pp. 226-243, 2008.

[27] C. Lin, S. Chen S, L. Yang, X. Bai, and Y. Li, "Effect of staphylococcal enterotoxin A on the distribution and clonal expansion of TCRV beta subfamilies and the cytotoxicity of T cells stimulated by PML-RAR alpha peptid," Blood, vol. 110, article 3871, 2007.

[28] U. Holzer, T. Orlikowsky, C. Zehrer et al., "T-cell stimulation and cytokine release induced by staphylococcal enterotoxin A (SEA) and the SEAD227A mutant," Immunology, vol. 90, no. 1, pp. 74-80, 1997.

[29] O. Dauwalder, A. Pachot, M. A. Cazalis et al., "Early kinetics of the transcriptional response of human leukocytes to staphylococcal superantigenic enterotoxins A and G," Microbial Pathogenesis, vol. 47, no. 3, pp. 171-176, 2009.
[30] K. M. Brauer, D. Werth, K. von Schwarzenberg et al., "BCR$\mathrm{ABL}$ activity is critical for the immunogenicity of chronic myelogenous leukemia cells," Cancer Research, vol. 67, no. 11, pp. 5489-5497, 2007.

[31] E. Wallace and L. Gewin, "Imatinib: novel treatment of immune-mediated kidney injury," Journal of the American Society of Nephrology, vol. 24, no. 5, pp. 694-701, 2013.

[32] N. Akashi, I. Matsumoto, Y. Tanaka et al., "Comparative suppressive effects of tyrosine kinase inhibitors imatinib and nilotinib in models of autoimmune arthritis," Modern Rheumatology, vol. 21, no. 3, pp. 267-275, 2011.

[33] G. S. Vratsanos, S. Jung, Y. M. Park, and J. Craft, "CD4+ T cells from lupus-prone mice are hyperresponsive to $\mathrm{T}$ cell receptor engagement with low and high affinity peptide antigens: a model to explain spontaneous T cell activation in lupus," Journal of Experimental Medicine, vol. 193, no. 3, pp. 329-337, 2001.

[34] E. C. Jury, P. S. Kabouridis, A. Abba, R. A. Mageed, and D. A. Isenberg, "Increased ubiquitination and reduced expression of LCK in T lymphocytes from patients with systemic lupus erythematosus," Arthritis and Rheumatism, vol. 48, no. 5, pp. 1343-1354, 2003.

[35] W. Wang, X. Sun, L. Lu, J. B. Zheng, Y. Tian, and W. Wang, "Cytotoxicity of lymphocytes activated by superantigen toxicshock-syndrome toxin-1 against colorectal cancer LoVo cells," Molecular and Cellular Biochemistry, vol. 376, no. 1-2, pp. 11-20, 2013.

[36] A. Sundstedt, M. Celander, H. Eriksson, M. Törngren, and G. Hedlund, "Monotherapeutically nonactive CTLA-4 blockade results in greatly enhanced antitumor effects when combined with tumor-targeted superantigens in a B16 melanoma model," Journal of Immunotherapy, vol. 35, no. 4, pp. 344-353, 2012.

[37] M. Kato, Y. Nakamura, T. Suda et al., "Enhanced anti-tumor immunity by superantigen-pulsed dendritic cells," Cancer Immunology, Immunotherapy, vol. 60, no. 7, pp. 1029-1038, 2011.

[38] G. Forsberg, L. Ohlsson, T. Brodin et al., "Therapy of human non-small-cell lung carcinoma using antibody targeting of a modified superantigen," British Journal of Cancer, vol. 85, no. 1, pp. 129-136, 2001.

[39] M. Dohlsten, G. Hedlund, E. Akerblom, P. A. Lando, and T. Kalland, "Monoclonal antibody-targeted superantigens: a different class of anti-tumor agents," Proceedings of the National Academy of Sciences of the United States of America, vol. 88, no. 20, pp. 9287-9291, 1991.

[40] D. W. Newton, M. Dohlsten, P. A. Lando, T. Kalland, C. Olsson, and M. Kotb, "MHC class II-independent, Vbetaspecific activation of $\mathrm{T}$ cells by superantigen mutants fused to anti-tumor Fab fragments: implications for use in treatment of human colon carcinoma," International Journal of Molecular Medicine, vol. 1, no. 1, pp. 157-162, 1998.

[41] J. Kappler, B. Kotzin, L. Herron et al., "V $\beta$-specific stimulation of human T cells by staphylococcal toxins," Science, vol. 244, no. 4906, pp. 811-813, 1989.

[42] D. Thomas, O. Dauwalder, V. Brun et al., "Staphylococcus aureus superantigens elicit redundant and extensive human $\mathrm{V} \beta$ patterns," Infection and Immunity, vol. 77, no. 5, pp. 2043-2050, 2009.

[43] N. Pumphrey, A. Vuidepot, B. Jakobsen, G. Forsberg, B. Walse, and K. Lindkvist-Petersson, "Cutting edge: evidence of direct TCR $\alpha$-chain interaction with superantigen," Journal of Immunology, vol. 179, no. 5, pp. 2700-2704, 2007.

[44] A. Herman, G. Croteau, R. P. Sekaly, J. Kappler, and P. Marrack, "HLA-DR alleles differ in their ability to present staphylococcal 
enterotoxins to T cells," Journal of Experimental Medicine, vol. 172, no. 3, pp. 709-717, 1990.

[45] W. Fujimaki, M. Iwashima, J. Yagi et al., "Functional uncoupling of T-cell receptor engagement and Lck activation in anergic human thymic CD4+ T cells," Journal of Biological Chemistry, vol. 276, no. 20, pp. 17455-17460, 2001.

[46] C. Bueno, C. D. Lemke, G. Criado et al., "Bacterial superantigens bypass Lck-dependent T cell receptor signaling by activating a G $\alpha 11$-dependent, PLC- $\beta$-mediated pathway," Immunity, vol. 25, no. 1, pp. 67-78, 2006.

[47] R. Zamoyska, “Superantigens: supersignalers?” Science's STKE, vol. 2006, no. 358, article pe45, 2006.

[48] G. Criado and J. Madrenas, "Superantigen stimulation reveals the contribution of Lck to negative regulation of T cell activation," Journal of Immunology, vol. 172, no. 1, pp. 222-230, 2004.

[49] L. I. R. Ehrlich, P. J. R. Ebert, M. F. Krummel, A. Weiss, and M. M. Davis, "Dynamics of p56lck translocation to the T cell immunological synapse following agonist and antagonist stimulation," Immunity, vol. 17, no. 6, pp. 809-822, 2002.

[50] M. Bocchia, E. Abruzzese, F. Forconi et al., "Imatinib does not impair specific antitumor T-cell immunity in patients with chronic myeloid leukemia," Leukemia, vol. 20, no. 1, pp. 142-143, 2006. 


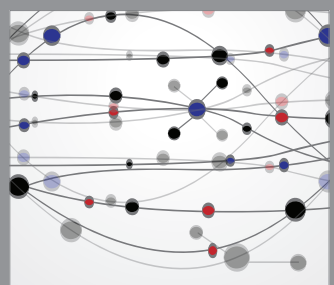

The Scientific World Journal
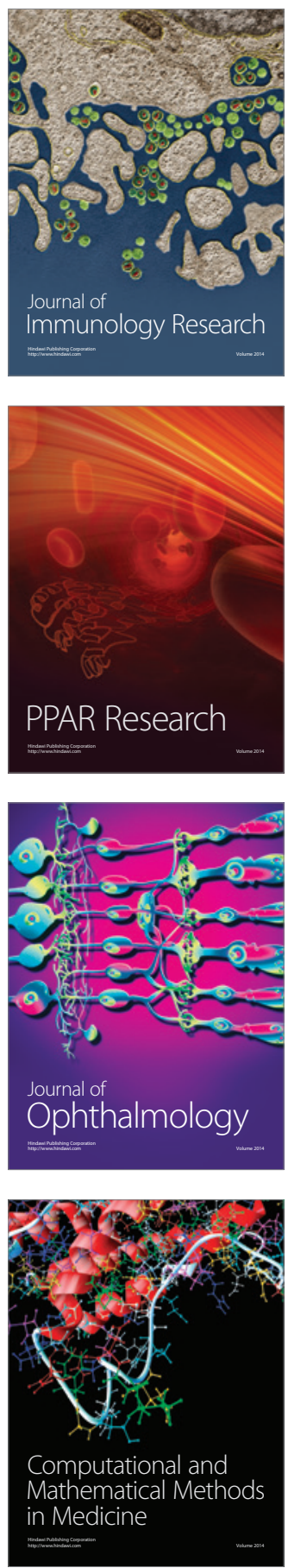

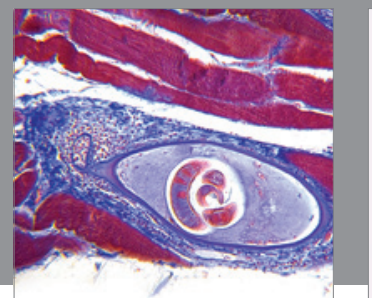

Gastroenterology

Research and Practice
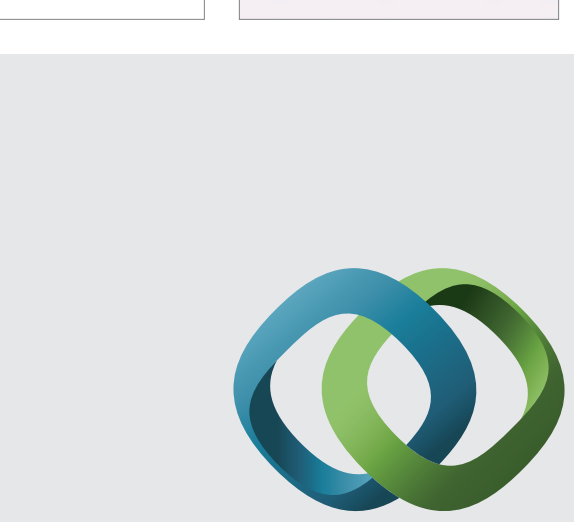

\section{Hindawi}

Submit your manuscripts at

http://www.hindawi.com
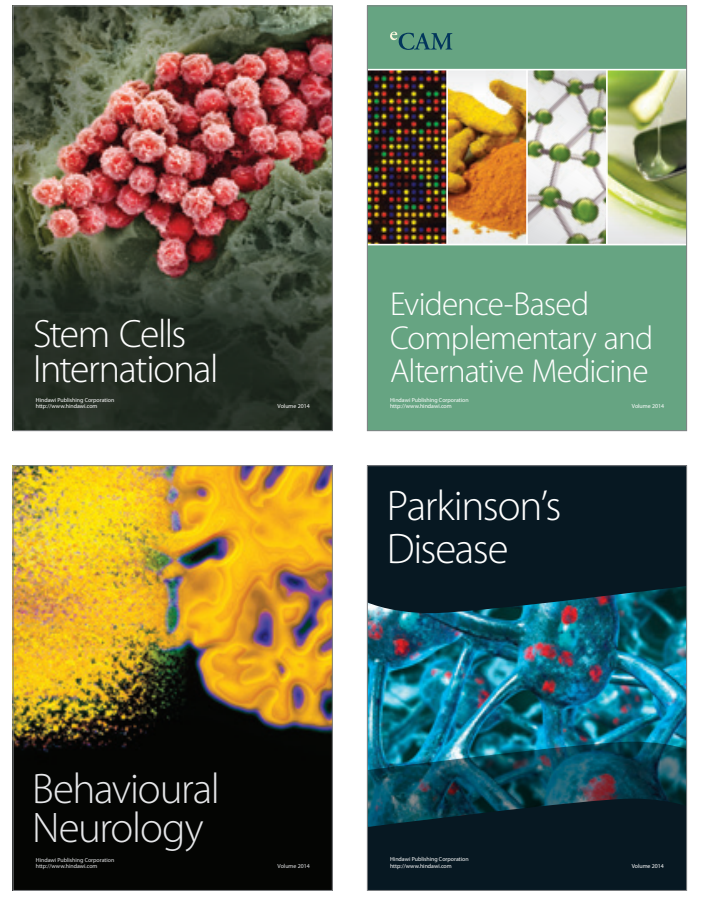
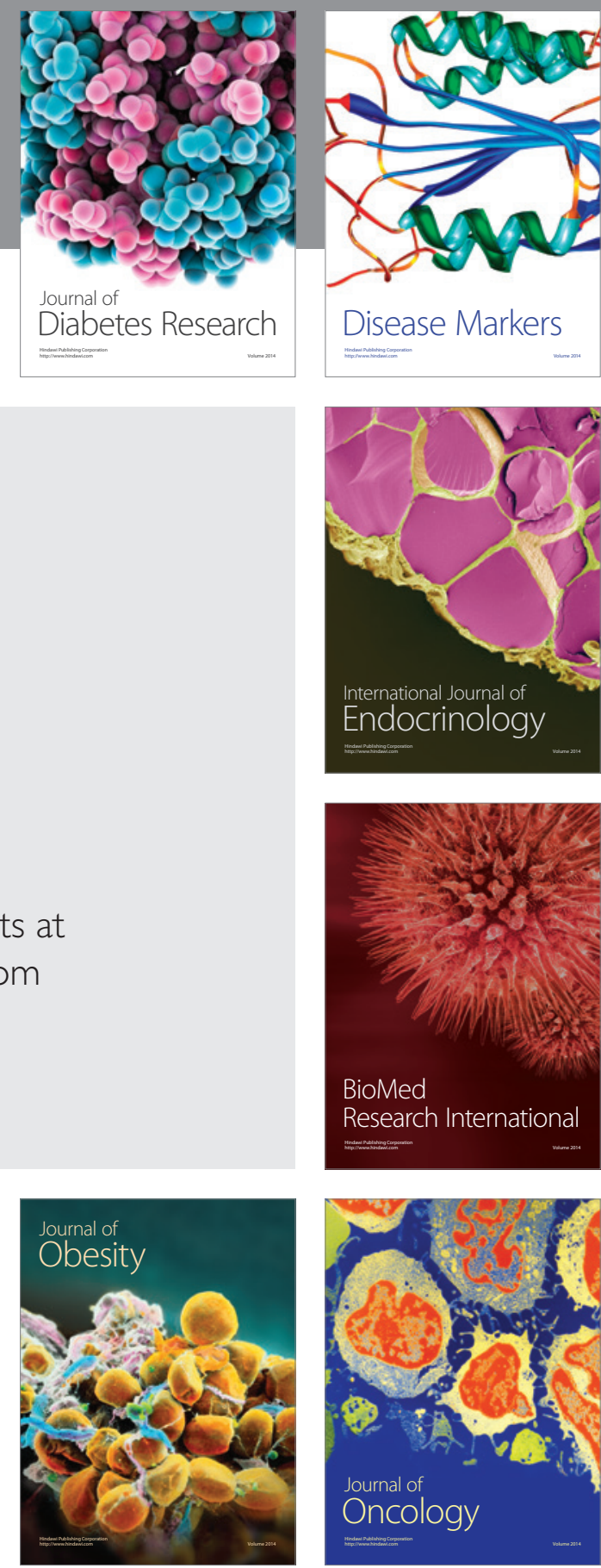

Disease Markers
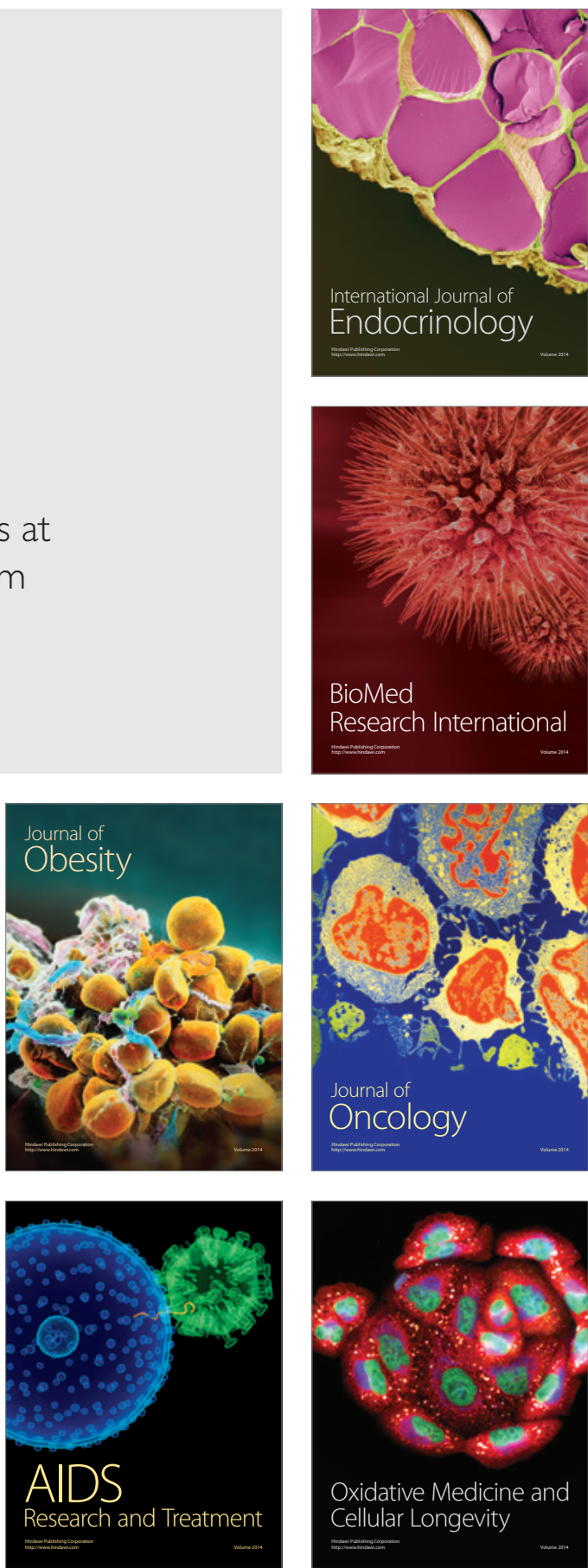